\title{
Enseñar a pensar en las posibilidades
}

\section{Teaching to think about the possibilities}

\author{
J. Eduardo Sierra Nieto*
}

Recibido: 14 de julio de 2020 Aceptado: 15 de julio de 2020 Publicado: 31 de julio de 2020

To cite this article: Sierra, J. E. (2020). Enseñar a pensar en las posibilidades. Márgenes, Revista de Educación de la Universi-

dad de Málaga, 1 (2), 175-179

DOI: https://doi.org10.24310/mgnmar.v1i2.9901

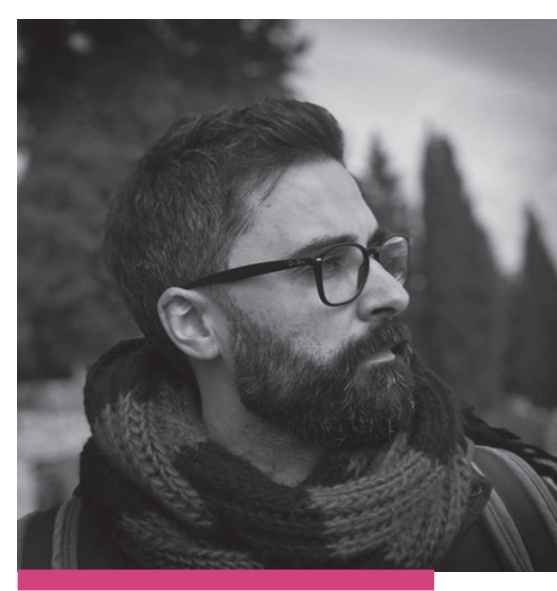

J. Eduardo Sierra Nieto

\section{RESUMEN}

Ser guía o acompañante se ha convertido en un cliché en el mundo educativo, también en las clases de formación del profesorado. Sin embargo, el mero hecho de proponernos como maestras y maestros un lugar mediador no resuelve sin más la situación. Se hace preciso explorar esa idea de mediación e ir dando forma a un lugar propio en la enseñanza. En este texto, el autor se detiene en un aspecto concreto: si enseñar quiere decir mostrar y no adoctrinar ¿la función de una maestra o de un maestro consiste en "mostrar todas las posibilidades”, o en abrir la posibilidad de "pensar en las posibilidades"?

Palabras clave: oficio docente; acompañamiento; mediación; adoctrinamiento; influencia; pensar

\section{ABSTRACT}

Being a guide or companion has become a cliche in the educational world, also in teacher education. However, the mere fact of proposing ourselves as teachers a mediating place does not resolve the situation. It is necessary to explore this idea of mediation and to create its own meaning in teaching. In this text, the author reflects on a specific aspect: if teaching means to show and not to indoctrinate, does the function of a teacher consist in "showing all the possibilities", or in opening the possibility of "thinking about the posibilities"?

Keywords: teaching profession; educational accompaniment; cultural mediation; indoctrinate; influence; thinking

"J. Eduardo Sierra Nieto http://orcid.org/0000-0002-9925-1656

Departamento de Teoría e Historia de la Educación y MIDE, Facultad de Educación, Universidad de Málaga esierra@uma.es

Twitter: @edukrator

https://uma.academia.edu/JEduardoSierraNieto 


\section{H I S T O R I A S M Í N I M A S}

En la educación infantil, visualizar a las maestras y a los maestros como guías y acompañantes se ha convertido en una figura recurrente, cuando no en un cliché. Algo que aprecio también en las aulas universitarias. Bajo esa imagen se nos representa una relación decididamente antiautoritaria que reclama, consecuentemente, ir dando forma a otra en la que lo primordial del oficio educativo (la mediación con la cultura y la entrada en el mundo de lo simbólico) no quede desdibujado.

Lo que ocurre, a mi modo de ver, es que solemos ir dando bandazos entre ese autoritarismo que rechazamos de plano y su aparente opuesto: ponernos del lado de nuestro alumnado. Pero la educación no consiste en decidir si estamos en contra o a favor de las niñas y de los niños, sino en recrear y sostener — con originalidad y apertura - el a favor.

La tendencia a resolver el asunto por la vía de un $a$ favor sin condiciones, es algo que identifico en muchos de los trabajos de clase. En estos, aparecen afirmaciones que van en la línea de "promover relaciones simétricas con el alumnado" o entender a "profesores y estudiantes como iguales”. Sin embargo, diría, la educación no es una cuestión de simetría o igualdad (al menos no en el sentido de coincidencia o correspondencia), sino de disparidad.

Lo anterior me lleva a pensar en que durante la formación es crucial dedicarnos a estudiar y comprender la naturaleza de la educación como experiencia de vinculación y, desde ahí, pensar la enseñanza como influencia respetuosa. A partir de ello, quiero proponer algunas reflexiones en torno a una de las opciones que parecen tomar mis estudiantes respecto de sulugar en la enseñanza, y que se sintetiza en la idea de que una maestra, un maestro, debe poder enseñar todas las posibilidades. Me explicaré.

Es habitual que alguna estudiante plantee que, frente a una enseñanza que solo muestra un punto de vista y por ello adoctrina, habría que proponer otra que muestre todos los puntos de vista, todas las posibilidades. En ese razonamiento, que reaparece cada curso, hay dos ideas que destacan.
"Pero la educación no consiste en decidir si estamos en contra o a favor de las niñas y de los niños, sino en recrear $y$ sostener -con originalidad y apertura- el $a$ favor." 


\section{"El asunto} me pareció interesante en al menos dos sentidos: porque nos invitaba a pensar en nuestro lugar en la mediación cultural y porque nos invitaba a pensar culturalmente en la idea de familia."
La primera es la aparente existencia de un constructo que podemos denominar como "todos los puntos de vista"; como si el conocimiento humano fuera una suma de partes que es posible ir recopilando y acumulando. La segunda tiene que ver con la imagen que se componen de un buen docente como aquel o aquella que, además de haberse cultivado en el conocimiento de "todos los puntos de vista", se mantiene al margens. En resumidas cuentas, y según esta perspectiva: un buen maestro, una buena maestra, es quien enseña todas las opciones sin revelar la suya.

En ese sentido, el curso pasado se nos presentó la ocasión de seguir pensando sobre ello, a propósito de uno de los temas estrella en educación infantil: los tipos de familia. Nos preguntamos: ¿cómo enseñar a las niñas y a los niños que existen distintos tipos de familias? Una conversación que se inició en buena medida porque está presente en nuestro día a día y, también, porque la ultraderecha se está encargando de revitalizar posiciones reaccionarias respecto de cómo las personas nos vinculamos y convivimos.

En el curso de la conversación, a muchas de las estudiantes les pareció muy razonable que circunscribir la enseñanza a mostrar como única opción posible la familia heteronormativa no era educativo; resultaba una posición sesgada y poco sensible con la realidad social. Hasta aquí, bien. Sin embargo, al pensar en al-

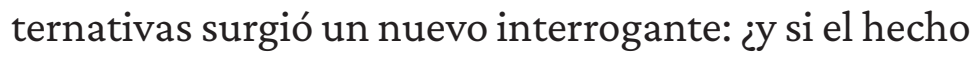
de elegir como maestras ${ }^{1}$ otros modelos de familia que mostrar en clase, acaba resultando también adoctrinador? Y desde aquí es que emergió la idea de "enseñar todos los modelos de familia" como la opción al parecer más adecuada.

El asunto me pareció interesante en al menos dos sentidos: porque nos invitaba a pensar en nuestro lugar en la mediación cultural y porque nos invitaba a pensar culturalmente en la idea de familia (esto sirve para cualquier otro contenido escolar).

1 Vengo utilizando el femenino pues me estoy refiriendo a las clases de Teoría de la Educación en el Grado de Educación Infantil (UMA), donde prácticamente el 100\% del alumnado son mujeres. 


\section{H I S T O R I A S M Í I M A S}

Respecto del lugar del docente, parecían tener problemas para aceptar que como maestras deberán posicionarse, y que esa toma de postura no es equivalente automáticamente a la manipulación o al adoctrinamiento. A ellas les preocupa que las niñas y los niños aprendan a pensar por sí mismos, y la postura acorde — bajo su razonamiento- es que para conseguirlo han de desaparecer como portadoras de una posición propia al respecto (nuevamente, esto sirve para cualquier otro contenido escolar). Pero quienes hemos pasado tiempo con niños y niñas, sabemos que su inclinación es la de buscar la autenticidad y reclamar nuestras verdades. Esto es, nos preguntan a menudo para saber qué pensamos, cómo vemos las cosas, cómo las experimentamos. Así es como van haciéndose una idea de nuestras opciones en el camino por dar forma a las suyas.

¡Claro que una maestra o un maestro debe custodiar la posibilidad de pensar por uno mismo! Pero sin que ese propósito acabe obturando el hecho de que la libertad de pensamiento tiene que ver con la vinculación y la mediación adulta, y no con la autosuficiencia.

En cuanto a pensar culturalmente me estoy refiriendo a que lo importante no es tanto qué opción es la propia (algo por otro lado crucial) sino el hecho de que las maestras y los maestros deben saber explorar los asuntos humanos desde un punto de vista culturalmente rico para así dar que pensar en la escuela.

Frente a la tesitura descrita, les preguntaba: ¿habéis pensado qué es una familia, más allá de los lugares comunes? ¿Creéis que es un concepto inmutable a lo largo y ancho del mundo? ¿Cuál es vuestra experiencia de la vida en familia? ¿Un amigo puede ser considerado como un familiar? Un señor entrado en los cincuenta que comparte su día a día con su gato ¿tiene una familia? Y una anciana viuda que acoge estudiantes en programas universitarios de alojamiento ¿la tiene?

$\mathrm{Al}$ principio no parecían entender qué sentido podrían tener esas preguntas, pues para ellas la cuestión estaba bien clara: frente a una enseñanza aparentemente parcial que barre para su terreno, otra completista que muestra el abanico entero.

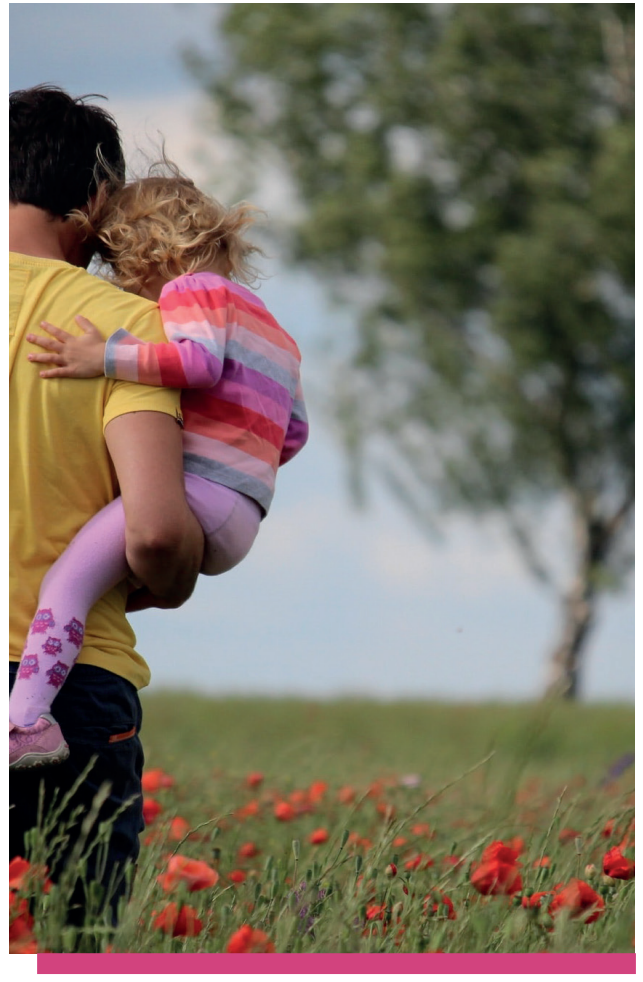

Photo by Juliane Liebermann on Unsplash 


\section{H I S T O R I S M Í N I M A S}

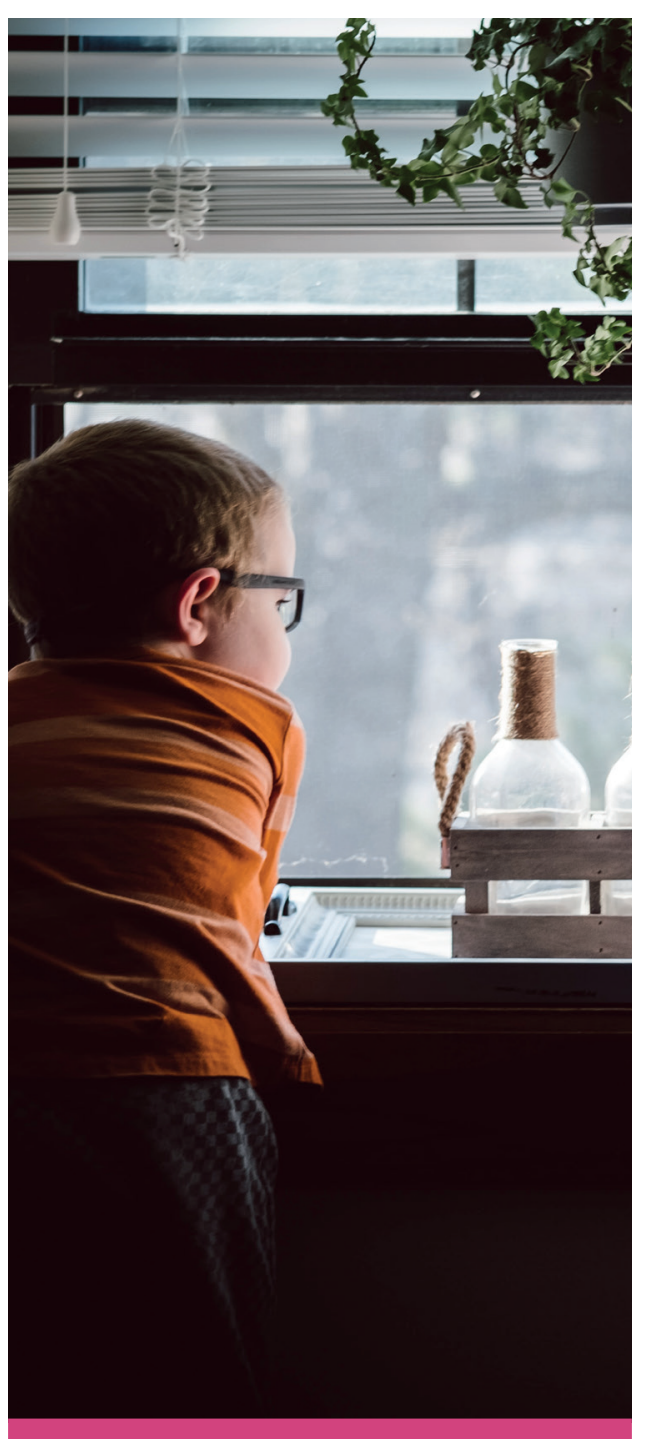

Photo by Andrew Seaman on Unsplash
Durante un tiempo exploramos esas y otras preguntas, y descubrimos que, si bien reconocíamos elementos comunes, sus experiencias en familia revelaban sentidos vitales dispares. Aparecían historias y sentires que nos hablaban, no ya de "los tipos de familia” sino de la vida en familia.

Poco a poco, la conversación vehiculaba un aprendizaje aperturista, de tal manera que... ¡estábamos aprendiendo acerca de los tipos de familia! Solo que lo hacíamos desde la narración de vivencias que nos permitían reconocer y respetar distintas opciones vitales; explorando también la complejidad - y la riquezaque ha entrañado y entraña organizar la vida en común.

Y así fue como pasamos del "enseñar todas las posibilidades” al "pensar en la posibilidad de éstas”. Todo un juego de palabras. Y todo un reto educativo. 